\title{
Effect of movement type on the classification of electromyography data for the control of dexterous prosthetic hands
}

\author{
Manfredo Atzori, Elisa Rosanda, Giorgio Pajardi, Franco Bassetto, Henning Müller
}

\begin{abstract}
Hand amputations can dramatically affect the capabilities of a person. Machine learning is often applied to Surface Electromyography (sEMG) to control dexterous prosthetic hands. However, it can be affected by low robustness in real life conditions, mainly due to data variability depending on various factors (such as the position of the limb, of the electrodes or the characteristics of the subject).

This paper aims at improving the understanding of sEMG for prosthesis control introducing the type of hand movement as a variable that influences classification performance in both intact subjects and hand amputees.

Five hand amputees and five matched intact subjects were selected from the publicly available NinaPro database. The subjects were recorded while repeating 40 hand movements. Movement classification was performed on the SEMG data with a window-based approach (concatenating several signal features) and a Random Forest classifier. The results show that some hand movements are classified significantly better than others $(\mathbf{p}<0.001)$ and there is a correspondence in how well the same hand movements are classified in intact subjects and hand amputees.

This work leads to advancements in the domain, highlighting the importance of the acquisition protocol for SEMG studies and suggesting that specific movements can lead to better performance for the control of prosthetic hands.
\end{abstract}

\section{INTRODUCTION}

An upper limb amputation is a major injury that can strongly affect the daily life of a person. Dexterous, natural control of robotic prostheses via machine learning and surface electromyography (sEMG) is promising but difficult to reach in real life. The most advanced control systems developed in scientific research are still far from being comparable to the natural control of a real hand and they often lack robustness in complex everyday conditions.

In 2005, the number of people living in the United States with a loss of an upper-limb was 41,000 [1]. The primary prosthetic upper-limb options are: cosmetic, body-powered, and myoelectric. Myoelectric prostheses are devices controlled via sEMG, usually using two muscular groups (respectively to close and open the prosthesis).

The use of machine learning techniques has improved the control of myoelectric prostheses in scientific research. These methods usually use a range of sEMG electrodes to record the myoelectric signals of the muscles that used to control the

\footnotetext{
*This work is partially supported by the Swiss National Science Foundation in the Sinergia project \#160837 MeganePro and by the Hasler Foundation in the project ProHand.

M. Atzori and H. Müller are with are with the Information Systems Institute, University of Applied Sciences Western Switzerland (HES-SO Valais), Sierre, Switzerland. (e-mail: manfredo.atzori@hevs.ch).
}

hand. The sEMG activity is analyzed using algorithms for pattern recognition to classify the movements that the subject intends to do [2]. In a virtual environment, a good representation of prosthesis usability was also obtained by performing real-time tests [2], [3]. Adapting a user's action to the system in a real-time user-machine interaction can help reduce some limitations [4]. On the other hand, offline analysis has several advantages for preliminary studies and benchmarking methods. Recently, additional sources of information [4]-[12] were proposed to be included in addition to sEMG, including for instance accelerometry, computer vision and eye tracking.

In few cases, the control advancements obtained by scientific researchers in this domain have been translated to commercial solutions. In 2013, Coapt-engineering1 made a new hand prosthesis control system based on real-time pattern recognition. Despite the strong improvement, these systems still control few movements and do not replicate the natural control of a biological hand [13].

When dealing with real life conditions, sEMG control systems based on machine learning are often affected by low robustness, also due to variability depending on factors such as the position of the limb, the exact positioning of the electrodes, the clinical characteristics of the subject and even the previous use of sEMG prostheses [13]-[15]. The positioning of the limb can influence the recorded sEMG activity. The effect of this phenomenon on the classification of hand movements using machine learning was investigated in several studies, together with means to solve it [16]-[20]. Various clinical parameters (including remaining forearm percentage and phantom limb sensation) strongly influence sEMG control performance [21], thus classification accuracy depends also on the characteristics of each amputee.

Hand movements have been widely studied in scientific literature. Taxonomies of hand movements tried to group them on the basis of qualitative (e.g. [22]) and, more recently, quantitative parameters [23]. The scientific literature on muscular and kinematic hand synergies also shows that a limited number of time-dependent motor modules may underlie the execution of a large variety of hand grasps [24][26]. The aim of this paper is to improve the understanding of sEMG variability introducing movements as a further variable affecting classification performance. The results obtained on intact subjects and hand amputees are then compared in order

F. Bassetto is with the Clinic of Plastic Surgery, Padova University Hospital, Padova, Italy.

E. Rosanda and G. Pajardi are with Unità Operativa Complessa di Chirurgia della Mano, Università degli Studi di Milano, Ospedale San Giuseppe Milan /IRCCS MultiMedica, Milano, Italy.

1 http://www.coaptengineering.com/ 
to evaluate if there is any correspondence in how the movements are reproduced by the two groups.

\section{METHODS}

\section{A. Subjects}

The subjects analyzed in this experiment include 5 hand amputees and 5 intact subjects respectively from the 2 nd and 3rd NinaPro dataset [27]. The subjects were matched by gender, age, height and weight in order to reduce possible differences in performance among the groups. All the subjects are males and the age, weight and height are reported for each subject in Table I. Average age is (38.2 \pm 5.8$)$ years for hand amputees and $(35.4 \pm 5.59)$ years for intact subjects; average height is $(177.4 \pm 7.2) \mathrm{cm}$ for hand amputees and $(176 \pm 9.02) \mathrm{cm}$ for intact subjects; average weight is $(79.8 \pm 9.9) \mathrm{Kg}$ for hand amputees and $(77.8 \pm 4.21) \mathrm{Kg}$ for intact subjects.

\section{B. Acquisition setup}

The acquisition setup includes several sensors (designed to record sEMG and hand kinematics) that are connected to a laptop performing the data acquisitions. The myoelectric activity of the forearm was recorded with a Delsys Trigno Wireless sEMG System. The system is composed of 16 sEMG electrodes, each containing a three- axial accelerometer. The
sEMG data are sampled at $2 \mathrm{kHz}$. The baseline noise is indicated as less than $0.5 \mathrm{mV}$ RMS [27]. The described locations were chosen in order to combine a dense sampling approach [28]-[30] and precise anatomical positioning strategy [31], [32]. Eight electrodes are placed around the forearm at equal distance from each other, at the height of the radio-humeral joint. Four electrodes are placed at the main activity spots (identified by palpation) of the flexor digitorum superficialis, of the extensor digitorum superficialis, of the biceps brachii and of the triceps brachii. The acquisition setup is described in detail in Atzori et al. [27].

TABLE I. CHARACTERISTICS OF THE SUBJECTS

\begin{tabular}{|c|c|c|c|c|c|c|}
\hline Age & Height & Weight & $\begin{array}{c}\text { Missing } \\
\text { limb }\end{array}$ & $\begin{array}{c}\text { Remaining } \\
\text { forearm } \\
\text { percentage }\end{array}$ & $\begin{array}{c}\text { Years since } \\
\text { amputation }\end{array}$ & $\begin{array}{c}\text { Cause of } \\
\text { amputation }\end{array}$ \\
\hline 35 & 172 & 79 & None & N.A. & N.A. & N.A. \\
\hline 45 & 173 & 73 & None & N.A. & N.A. & N.A. \\
\hline 34 & 173 & 84 & None & N.A. & N.A. & N.A. \\
\hline 32 & 170 & 75 & None & N.A. & N.A. & N.A. \\
\hline 31 & 192 & 78 & None & N.A. & N.A. & N.A. \\
\hline 35 & 183 & 81 & Left & 70 & 6 & Accident \\
\hline 34 & 166 & 68 & Right & 40 & 1 & Accident \\
\hline 33 & 175 & 80 & Right & 50 & 5 & Accident \\
\hline 44 & 180 & 95 & Right & 90 & 14 & Accident \\
\hline 45 & 183 & 75 & Right & 90 & 5 & Cancer \\
\hline
\end{tabular}

N.A. stands for "Not Admitted"

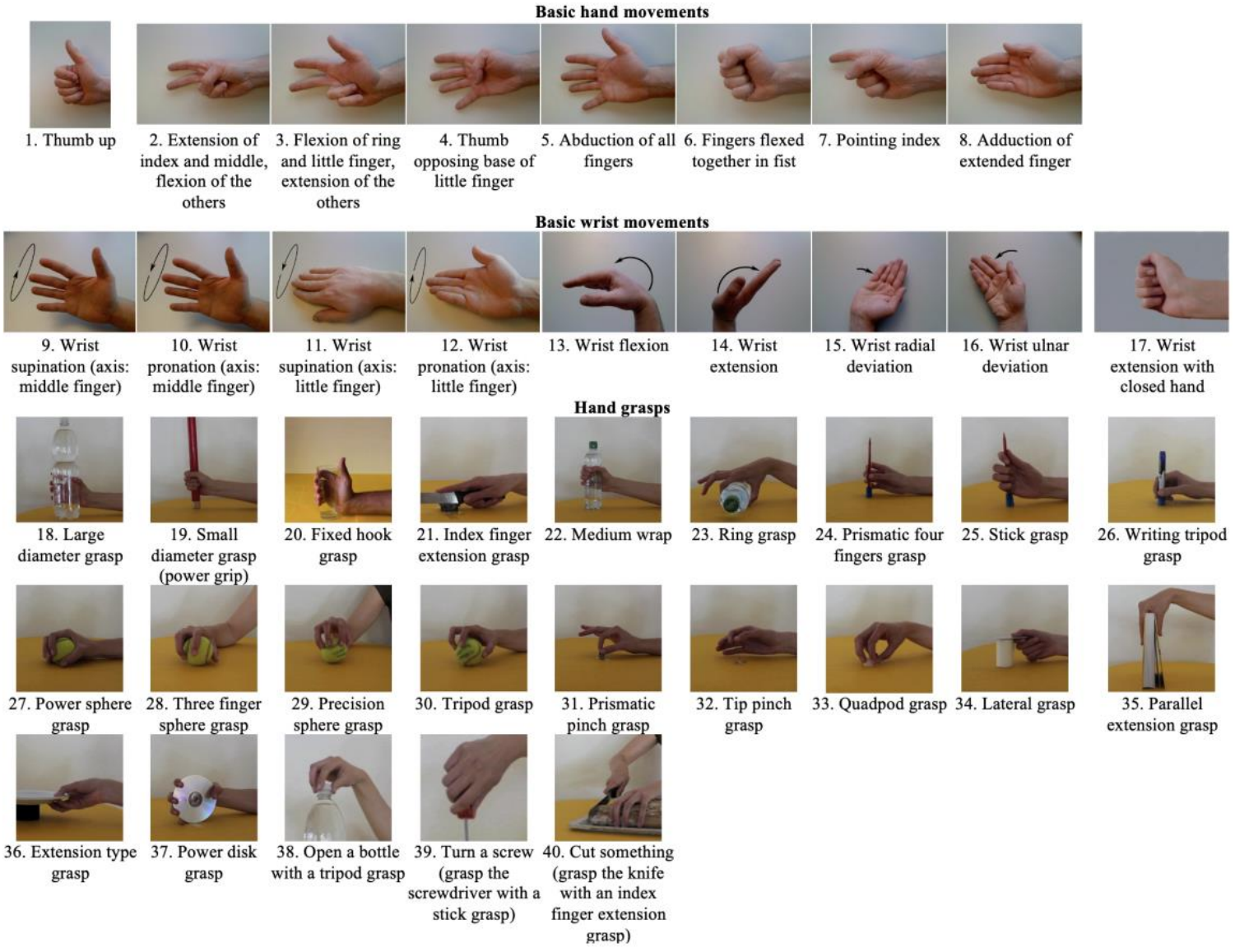

Figure 1 Hand movements included in the data analysis. 

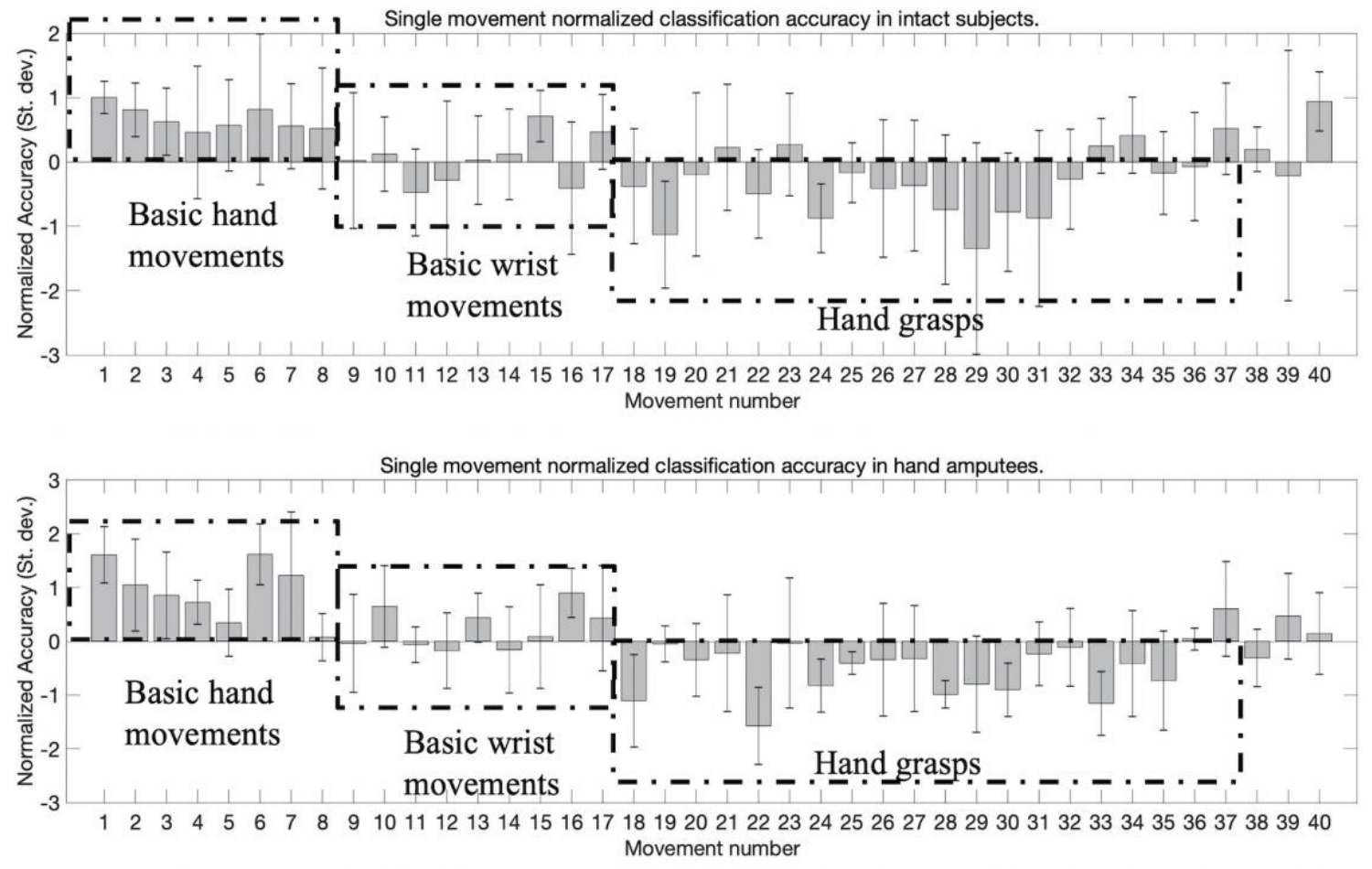

Figure 2 Average normalized classification accuracy of each hand movement (expressed in standard deviations from the average classification accuracy) for intact subjects (top) and hand amputees (bottom).

\section{Acquisition protocol}

Before the experiment, each subject was requested to sign an informed consent form and to answer a set of questions including age, gender, height, weight and laterality. Afterwards, subjects were asked to sit at a desk on an office chair while resting their arms on a desktop. In front of them, visual stimuli were provided via a laptop that also recorded data from the sensors. The experiment included a training session (consisting of a condensed mix of movements), and several exercises addressing different types of movements. Some minutes of rest were included between the exercises in order to reduce muscular fatigue. The movements considered in this experiment are 40 in total and they were selected from the most recent hand taxonomy and robotics literature [33][38] in order to permit most of the activities of daily living (ADL). The considered hand movements correspond to the NinaPro exercise A and B [27] and, as represented in Fig.1, they are divided into: basic hand movements (movement 1-8); basic wrist movement (movement 9-17); hand grasps (movement 18-40). Each movement was repeated 6 times, each repetition lasting 5 seconds and being followed by 3 seconds of rest. The subjects repeated the movements with the right hand, amputees repeated them with the missing limb as naturally as possible. Since the main aim of the data was to permit movement classification, all the subjects were asked to concentrate on mimicking the movements rather than on exerting high forces [21], [39], [40].

\section{Hand movement classification based on $S E M G$}

Signal pre-processing was performed before classification. Power line interference was filtered to avoid power line interference ( $50 \mathrm{~Hz}$ and harmonics) using a Hampel filter. The generalized likelihood ratio algorithm was used to correct imperfect labeling, mainly originating from the fact that the subjects may not perfectly mirror the movements shown on screen (also due to reaction times) [27].

The movement classification procedure follows Englehart et al. [17]. Each movement repetition is divided in time windows of $200 \mathrm{~ms}$; each window is labeled with its movement number, obtained after relabeling. Signal features were computed using PaWFE2, a publicly available signal feature extraction tool that allows to extract widely used features in short time using parallel time windows [41]. The analysed signal features consist of the normalized concatenation of: Root-Mean-Square (RMS), time domain statistics (TD) [42], Histogram (HIST) [43], marginal Discrete Wavelet Transform (mDWT) [44]. 
The used classifier is a Random Forest [45], with a total of 100 trees. Repetitions 1, 3, 4 and 6 were used for training, repetitions 2 and 5 were used for testing. This approach was used in several articles in the past and demonstrated to provide reasonable estimates while also having low computational costs [27], [46]. The classification was performed on all movements.

Classification accuracy was normalized subtracting the sample average and dividing by the standard deviation. The Kruskal-Wallis test, Pearson's correlation and Deming regression were used to perform statistical evaluation of the results [47], [48].

\section{RESULTS}

The type of hand movement influences the classification accuracy and there is a correspondence in how well the same hand movements are classified in intact subjects and hand amputees (Fig. 2, 3).

Some movements are classified significantly better than others and this is highlighted when considering normalized classification accuracy. Classification accuracy varies (depending on the movement) both in intact subjects and hand amputees. In intact subjects, the average movement-specific classification accuracy ranges between $(54.65 \pm 24.44) \%$ and $(91.7 \pm 6.97) \%$. In transradial amputees, it ranges between $(23 \pm 18.49) \%$ and $(84.37 \pm 13.82) \%$. There are significant differences between the average classification accuracy of different movements, both in hand amputees and intact subjects (respectively $\mathrm{p}<0.001$ and $\mathrm{p}<0.05$ ). After normalizing the classification accuracy for each group, the differences among different movements get even stronger ( $p<0.001$ for both intact subjects and hand amputees) (Fig.2). Normalizing the classification accuracy allows also to identify overall classification accuracy patterns depending on the group of movements. In particular, the group "basic hand movements" is classified on average with higher normalized classification accuracy than the group "basic wrist movements", that has higher normalized classification accuracy than "hand grasps". This pattern is followed both by intact subjects and hand amputees (Fig.2), as well as the fact that normalized classification accuracy of functional movements strongly depends on the movement.

There is a correspondence in how well each hand movement is classified in intact subjects and hand amputees. Deming regression (Fig. 3) allows to visualize the correspondence between the average normalized classification accuracy obtained for each movement in intact subjects and in hand amputees. Comparing the normalized classification accuracy obtained of each movement in intact subjects with the results obtained in hand amputees leads to no significant differences in any of the 40 movements considered. Pearson's correlation of the average normalized classification accuracies obtained for each movement in intact subjects with the accuracies obtained for hand amputees is equal to 0.64 . The pvalue obtained for the correlation using a Student's tdistribution is also below 0.001 , rejecting the hypothesis that no correlation exists between the average accuracy obtained for intact subjects and hand amputees.

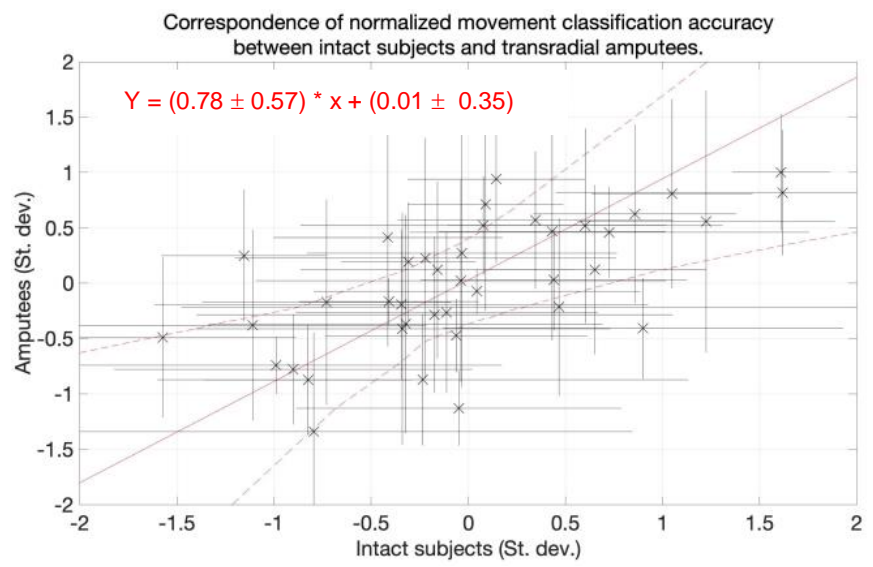

Figure 3 Correspondence of normalized movement classification accuracy between intact subjects and amputees. Deming regression of the normalized classification accuracy of each hand movement (expressed in standard deviations from the average classification accuracy). Confidence intervals represent 2.5 standard deviations.

\section{CONCLUSION}

The results described in this article bring several advancements to the domain of sEMG controlled dexterous robotic prosthetic hands.

First, they highlight that the movements included in the acquisition protocol influence the performance of the machine learning algorithms. Second, they suggest that specific movements can lead to better performance than others for the control of robotic prosthetic hands. Control robustness of prosthetic hands may thus be augmented also by developing control systems that are based on groups of movements that obtain higher average classification accuracy. The overall correspondence of movement-specific normalized classification accuracy between intact subjects and hand amputees might also be exploited to develop successful transfer learning strategies, allowing to train the algorithms more quickly and to make them more robust.

The reasons behind the significant differences of classification accuracy among movements can be due to many factors, including muscular anatomy but also movement execution variability (related for instance to the fact that grasps require the interaction with objects, which can be performed in many slightly different ways or exerting different forces). It is important to analyse these factors in further experiments.

Finally, the study provides further evidence that it is possible to group hand movement types according to a few parameters, in accordance with the literature on movement taxonomies [22] [23] and kinematic or muscular synergies [24]-[26].

As highlighted in the article, the importance of the movements included in the acquisition protocol appears to be relevant for all work in the domain. Further studies should thus be performed including variations of the movements considered, as well as keeping into account confounding variables. 


\section{ACKNOWLEDGMENT}

The authors would like to thank the Swiss National Science Foundation and the Hasler foundation that partially supported this work respectively via the Sinergia project \# 160837 MeganePro and the project ProHand, as well as the patients and subjects that voluntarily participated to data acquisition.

\section{REFERENCES}

[1] K. Ziegler-Graham, E. J. MacKenzie, P. L. Ephraim, T. G. Travison, and R. Brookmeyer, "Estimating the prevalence of limb loss in the United States: 2005 to 2050," Arch. Phys. Med. Rehabil., vol. 89, no. 3, pp. 422-429, 2008.

[2] E. Scheme and K. Englehart, "Electromyogram pattern recognition for control of powered upper-limb prostheses: State of the art and challenges for clinical use," J. Rehabil. Res. Dev., vol. 48, no. 6, p. 643, 2011.

[3] L. Hargrove, Y. Losier, B. Lock, K. Englehart, and B. Hudgins, "A real-time pattern recognition based myoelectric control usability study implemented in a virtual environment," Annu. Int. Conf. IEEE Eng. Med. Biol. - Proc., pp. 4842-4845, 2007.

[4] E. A. Corbett, K. P. Kording, and E. J. Perreault, "Real-Time evaluation of a noninvasive neuroprosthetic interface for control of reach," Neural Syst. Rehabil. Eng. IEEE Trans., vol. 21, no. 4, pp. 674-683, 2013.

[5] M. Markovic, S. Dosen, D. Popovic, B. Graimann, and D. Farina, "Sensor fusion and computer vision for context-aware control of a multi degree-of-freedom prosthesis.," J. Neural Eng., vol. 12, no. 6, p. 66022, 2015.

[6] M. Markovic, S. Dosen, C. Cipriani, D. Popovic, and D. Farina, "Stereovision and augmented reality for closed-loop control of grasping in hand prostheses," J. Neural Eng., vol. 11, no. 4, p. 46001, Aug. 2014

[7] M. Atzori, A. Gijsberts, H. Müller, and B. Caputo, "Classification of hand movements in amputated subjects by $\mathrm{sEMG}$ and accelerometers.," in Annual International Conference of the IEEE Engineering in Medicine and Biology Society (EMBC), 2014, p. 63.

[8] M. Cognolato et al., "Semi-automatic training of an object recognition system in scene camera data using gaze tracking and accelerometers," in International Conference on Computer Vision Systems (ICVS), 2017.

[9] F. Giordaniello et al., "obilMegane Pro: myo-electricity, visual and gaze tracking data acquisitions to improve hand prosthetics.," in IEEE International Conference on Rehabilitation Robotics, 2017.

[10] G. Saetta et al., "Gaze, behavioral, and clinical data for phantom limbs after hand amputation from 15 amputees and 29 controls," Sci. Data (Nature Publ. Group), vol. accepted, 2020.

[11] M. Cognolato et al., "Gaze, Visual, Myoelectric, and Inertial Data of Grasps for Intelligent Prosthetics," Sci. Data (Nature Publ. Group), vol. accepted, 2020.

[12] F. Giordaniello et al., "Megane Pro: myo-electricity, visual and gaze tracking integration as a resource for dexterous hand prosthetics," in IEEE International Conference on Rehabilitation Robotics, 2017.

[13] M. Atzori and H. Müller, "Control Capabilities of Myoelectric Robotic Prostheses by Hand Amputees: A Scientific Research and Market Overview," Front. Syst. Neurosci., vol. 9, no. 162, 2015.

[14] D. Farina et al., "The extraction of neural information from the surface EMG for the control of upper-limb prostheses: Emerging avenues and challenges," 2014.

[15] F. Bassetto, C. Tiengo, M. Atzori, and H. Müller, "Clinical, anatomical and external factors to improve dexterous robotic hand prostheses," in XXI Congress of the Federation of European Societies for Surgery of the Hand (FESSH), 2016.

[16] E. Scheme, A. Fougner, Stavdahl, A. D. C. Chan, and K. Englehart, "Examining the adverse effects of limb position on pattern recognition based myoelectric control," in 2010 Annual International Conference of the IEEE Engineering in Medicine and Biology Society, EMBC'10, 2010

[17] K. Englehart and B. Hudgins, "A robust, real-time control scheme for multifunction myoelectric control," IEEE Trans. Biomed.

Eng., vol. 50, no. 7, pp. 848-854, 2003.

[18] E. Scheme, K. Biron, and K. Englehart, "Improving myoelectric pattern recognition positional robustness using advanced training protocols," in Proceedings of the Annual International Conference of the IEEE Engineering in Medicine and Biology Society, EMBS, 2011.

[19] A. Fougner, E. Scheme, A. D. C. Chan, K. Englehart, O. Stavdahl, and Ø. Stavdahl, "Resolving the Limb Position Effect in Myoelectric Pattern Recognition," Neural Syst. Rehabil. Eng. IEEE Trans., vol. 19, no. 6, pp. 644-651, 2011.

[20] M. Cognolato, L. Brigato, Y. Dicente Cid, M. Atzori, and H. Müller, "Analyzing the trade-off between training session time and performance in myoelectric hand gesture recognition during upper limb movement," in IEEE International Conference on Rehabilitation Robotics (ICORR), 2019.

[21] M. Atzori et al., "Clinical Parameter Effect on the Capability to Control Myoelectric Robotic Prosthetic Hands," J. Rehabil. Res. Dev., vol. 53, no. 3, pp. 345-358, 2016.

[22] M. R. Cutkosky, "On grasp choice, grasp models, and the design of hands for manufacturing tasks," IEEE Trans. Robot. Autom., vol. 5, no. 3, pp. 269-279, 1989.

[23] F. Stival, S. Michieletto, M. Cognolato, E. Pagello, H. Müller, and M. Atzori, "A quantitative taxonomy of human hand grasps," J. Neuroeng. Rehabil., 2019.

[24] M. Santello et al., "Hand synergies: Integration of robotics and neuroscience for understanding the control of biological and artificial hands," Phys. Life Rev., vol. 17, pp. 1-23, Jul. 2016.

[25] A. Scano, A. Chiavenna, L. M. Tosatti, H. Müller, and M. Atzori, "Muscle synergy analysis of a hand-grasp dataset: A limited subset of motor modules may underlie a large variety of grasps," Front. Neurorobot., 2018.

[26] N. J. Jarque-Bou, A. Scano, M. Atzori, and H. Müller, "Kinematic synergies of hand grasps: A comprehensive study on a large publicly available dataset," J. Neuroeng. Rehabil., 2019.

[27] M. Atzori et al., "Electromyography data for non-invasive naturally-controlled robotic hand prostheses," Sci. Data, vol. 1, p. 140053, 2014.

[28] F. V. G. Tenore, A. Ramos, A. Fahmy, S. Acharya, R. EtienneCummings, and N. V Thakor, "Decoding of individuated finger movements using surface electromyography," IEEE Trans. Biomed. Eng., vol. 56, no. 5, pp. 1427-1434, May 2009.

[29] O. Fukuda, T. Tsuji, M. Kaneko, A. Otsuka, and O. F. T. Tsuji, "A human-assisting manipulator teleoperated by EMG signals and arm motions," IEEE Trans. Robot. Autom., vol. 19, no. 2, pp. 210-222, Apr. 2003.

[30] G. Li, A. E. Schultz, and T. A. Kuiken, "Quantifying pattern recognition-based myoelectric control of multifunctional transradial prostheses," in IEEE Trans Neural Syst Rehabil Eng, 2010, vol. 18, no. 2, pp. 185-192.

[31] C. J. De Luca, "The use of surface electromyography in biomechanics," J. Appl. Biomech., vol. 13, no. 2, pp. 135-163, 1997.

[32] C. Castellini, A. E. Fiorilla, and G. Sandini, "Multi-subject / daily-life activity EMG-based control of mechanical hands," $J$. Neuroeng. Rehabil., vol. 6, no. 41, 2009.

[33] N. Kamakura, M. Matsuo, H. Ishii, F. Mitsuboshi, and Y. Miura, "Patterns of static prehension in normal hands," Am. J. Occup. Ther. Off. Publ. Am. Occup. Ther. Assoc., vol. 34, no. 7, pp. 437445, 1980.

[34] M. R. Cutkosky, "On grasp choice, grasp models, and the design of hands for manufacturing tasks," IEEE Trans. Robot. Autom., vol. 5, no. 3, pp. 269-279, 1989.

[35] S. J. Edwards, D. J. Buckland, and J. D. McCoy-Powlen, Developmental and Functional Hand Grasps. Slack Incorporated, 2002.

[36] R. Kato, H. Yokoi, and T. Arai, "Competitive learning method for robust EMG-to-motion classifier," in Proceedings Intelligent Autonomus Systems, 2006, pp. 946-953.

[37] F. C. P. Sebelius, B. N. Rosen, and G. N. Lundborg, "Refined myoelectric control in below-elbow amputees using artificial neural networks and a data glove," J. Hand Surg. Am., vol. 30, no. 
4, pp. 780-789, 2005.

[38] B. Crawford, K. Miller, P. Shenoy, and R. Rao, "Real-Time Classification of Electromyographic Signals for Robotic Control," in Proceedings of AAAI, 2005, pp. 523-528.

[39] M. Atzori et al., "Characterization of a benchmark database for myoelectric movement classification," Trans. Neural Syst. Rehabil. Eng., 2014.

[40] M. Atzori et al., "Building the NINAPRO Database: A Resource for the Biorobotics Community," in Proceedings of the IEEE International Conference on Biomedical Robotics and Biomechatronics (BioRob), 2012, pp. 1258-1265.

[41] M. Atzori and M. Henning, "Pawfe: Fast signal feature extraction using parallel time windows," Front. Neurorobot., 2019.

[42] B. Hudgins, P. Parker, and R. N. Scott, "A new strategy for multifunction myoelectric control," Biomed. Eng. IEEE Trans., vol. 40, no. 1, pp. 82-94, 1993.

[43] M. Zardoshti-Kermani, B. C. Wheeler, K. Badie, and R. M. Hashemi, "EMG feature evaluation for movement control of upper extremity prostheses," IEEE Trans. Rehabil. Eng., vol. 3, no. 4, pp. 324-333, 1995.

[44] M. Lucas, A. Gaufriau, S. Pascual, C. Doncarli, and D. Farina, "Multi-channel surface EMG classification using support vector machines and signal-based wavelet optimization," Biomed. Signal Process. Control, vol. 3, no. 2, pp. 169-174, 2008.

[45] L. Breiman, "Random forests," Mach. Learn., vol. 45, pp. 5-32, 2001.

[46] S. Pizzolato, L. Tagliapietra, M. Cognolato, M. Reggiani, H. Müller, and M. Atzori, "Comparison of Six Electromyography Acquisition Setups on Hand Movement Classification Tasks," PLoS One, 2017.

[47] J. D. Gibbons and S. Chakraborti, Nonparametric Statistical Inference: Revised and Expanded. CRC press, 2014.

[48] W. E. Deming, "Statistical adjustment of data.," 1943. 